\title{
Retrospective Analysis of $S$ - 1 plus Bevacizumab as Maintenance Therapy after Induction of S-1 and Oxaliplatin (SOX) plus Bevacizumab as First-Line Chemotherapy in Patients with Metastatic Colorectal Cancer
}

\author{
Yasutake Uchima*, Naoki Aomatsu, Gen Tsujio, Takehiko Iwauchi, Shinji Matsutani, \\ Hiroto Tanaka, Hironari Miyamoto, Takuma Okada, Shigeaki Kurihara, Toshiki Hirakawa, \\ Keiichiro Hirata, Junya Morimoto, Shigehito Yamagata, Kazuhiro Takeuchi
}

Department of Surgery, Fuchu Hospital, Osaka, Japan

Email: *y_uchima@seichokai.or.jp

How to cite this paper: Uchima, Y., Aomatsu, N., Tsujio, G., Iwauchi, T., Matsutani, S., Tanaka, H., Miyamoto, H., Okada, T., Kurihara, S., Hirakawa, T., Hirata, K., Morimoto, J., Yamagata, S. and Takeuchi, K. (2020) Retrospective Analysis of S-1 plus Bevacizumab as Maintenance Therapy after Induction of S-1 and Oxaliplatin (SOX) plus Bevacizumab as First-Line Chemotherapy in Patients with Metastatic Colorectal Cancer. Journal of Cancer Therapy, 11, 35-43. https://doi.org/10.4236/jct.2020.112004

Received: November 27, 2019

Accepted: January 19, 2020

Published: January 22, 2020

Copyright $\odot 2020$ by author(s) and Scientific Research Publishing Inc. This work is licensed under the Creative Commons Attribution International License (CC BY 4.0).

http://creativecommons.org/licenses/by/4.0/

\section{(c) (i) Open Access}

\begin{abstract}
Background: The SOFT study was a phase III trial designed to validate the non-inferiority of S-1 and oxaliplatin (SOX) plus bevacizumab to mFOLFOX6 plus bevacizumab in terms of PFS in patients with metastatic colorectal cancer (mCRC) who had not previously received chemotherapy. In this study, we retrospectively reviewed cases in which S-1 plus bevacizumab as maintenance therapy after induction of S-1 and Oxaliplatin (SOX) plus bevacizumab as first-line chemotherapy in patients with metastatic colorectal cancer was applied in order to evaluate its efficacy and safety in clinical practice. Material and method: Among the 40 patients with mCRC at the Fuchu Hospital who received SOX plus bevacizumab as a first line treatment between August 2013 and December 2018. The eligible patients had histologically confirmed mCRC. On day 1 of each 3-week period during the study, patients in the SOX plus bevacizumab received a $7.5 \mathrm{mg} / \mathrm{kg}$ intravenous infusion of bevacizumab, followed by an intravenous infusion of $130 \mathrm{mg} / \mathrm{m}^{2}$ oxaliplatin. S-1 $(40-60 \mathrm{mg}$ ) was administered orally two times per day from after dinner on day 1 to after breakfast on day 15, followed by a 7-day rest. Results: The median PFS was 15.0 months and median OS was 36.0 months. The response rate (RR: complete pus partial response) was $85.0 \%$, and the disease control rate (DCR: RR plus stable disease) was $92.5 \%$. The most common adverse events with SOX plus bevacizumab were hypertension (50\%), neurosensory toxicity (50\%), anorexia (32.5\%), fatigue (45\%), pigmentation (39\%), Neutrophil count decreased (30\%),
\end{abstract}


and platelet count decreased (40\%). The most common grade $3 / 4$ adverse events were neurosensory toxicity (5\%) and fatigue (9\%). Conclusion: This study revealed that the survival benefit of S- 1 and oxaliplatin (SOX) plus bevacizumab in Japanese patients with mCRC was like that observed in previous clinical trials. Therefore, S- 1 and oxaliplatin (SOX) plus bevacizumab can be considered as routine first-line treatment option for patients with mCRC.

\section{Keywords}

S-1, Oxaliplatin, Bevacizumab, Colorectal Cancer

\section{Introduction}

Colorectal cancer is the third most frequent tumor in the world, with one million new cases being diagnosed every year [1]. In Japan, colorectal cancer is the first most common cancer and the second most common cause of death [2]. In metastatic colorectal cancer, doublet combination chemotherapy plus targeted agents (anti-VEGF antibody or anti-EGF receptor [EGFR] antibody) are widely accepted as first-line treatment. Combination treatment with 5-fluorouracil (5-FU), leucovorin (LV) and oxaliplatin (FOLFOX) plus bevacizumab is one of the standard therapies for mCRC [3] [4]. However, to administer FOLFOX, patients have to return to the hospital once every 2 weeks and require a central venous (CV) catheter and portable infusion pump. A CV catheter and infusion pump negatively affect the quality of life (QOL) of patients and can cause adverse events such as infection, thrombosis and various catheter-related problems. Capecitabine and oxaliplatin (CapeOX) regimen requires still fewer planned hospital visits, with oxaliplatin administered every 3 weeks and capecitabine taken orally. The NO16966 trial showed that CapeOX is noninferior to FOLFOX4 in terms of progression-free survival (PFS) as a first-line treatment for mCRC [5] [6]. Capecitabine is another oral fluoropyrimidine derivative, and patients do not require placement of a $\mathrm{CV}$ catheter.

We retrospectively reviewed cases in which XELOX (CapeOX) plus bevacizumab was applied in order to evaluate its efficacy and safety in clinical practice. We considered that XELOX (CapeOX) plus bevacizumab can be considered as routine first-line treatment option for patients with mCRC [7].

S-1 is an oral anticancer agent that combines tegafur (a prodrug of fluorouracil) with two modulators: gimeracil, which reversibly inhibits dihydropyrimidine dehydrogenase (the primary metabolizingenzyme of fluorouracil) and thereby maintains effective fluorouracil concentrations in the blood for prolonged periods; and oteracil potassium, which suppresses the activity and toxicity of fluorouracil in gastrointestinal tissue [8].

The SOFT study was a phase III trial designed to validate the non-inferiority of S-1 and oxaliplatin (SOX) plus bevacizumab to mFOLFOX6 plus bevacizumab in terms of PFS in patients with $\mathrm{mCRC}$ who had not previously received che- 
motherapy. SOX plus bevacizumab is non-inferior to mFOLFOX6 plus bevacizumab in terms of PFS in patients with mCRC who had not previously received chemotherapy. The median survival time (MST) was about 30 months and was similar in the SOX plus bevacizumab group and the mFOLFOX6 plus bevacizumab group. SOX plus bevacizumab is considered an effective regimen for first-line chemotherapy in patients with mCRC and can be used instead of mFOLFOX6 plus bevacizumab [9].

In this study, we retrospectively reviewed cases in which S-1 plus bevacizumab as maintenance therapy after induction of S- 1 and Oxaliplatin (SOX) plus bevacizumab as first-line chemotherapy in patients with metastatic colorectal cancer was applied in order to evaluate its efficacy and safety in clinical practice.

\section{Patients and Methods}

\subsection{Patients}

Among the 40 patients with mCRC at the Fuchu Hospital who received SOX plus bevacizumab as a first line treatment between August 2013 and December 2018. The eligible patientshad histological confirmed mCRC. The other inclusion criteria included an Eastern Cooperative Oncology Group (ECOG) performance status of $0-1$ and adequate hematological, liver, and renal functions. This study is a retrospective study and had no exclusion criteria.

\subsection{Treatment}

On day 1 of each 3-week period during the study, patients in the SOX plus bevacizumab received a $7.5 \mathrm{mg} / \mathrm{kg}$ intravenous infusion of bevacizumab, followed by an intravenous infusion of $130 \mathrm{mg} / \mathrm{m}^{2}$ oxaliplatin. S-1 $[40-60 \mathrm{mg}$, based on the body surface area (BSA): BSA $<1.25 \mathrm{~m}^{2}, 40 \mathrm{mg}$; BSA $\geq 1.25 \mathrm{~m}^{2}$ to $<1.5 \mathrm{~m}^{2}, 50$ $\mathrm{mg}$; BSA $\geq 1.5 \mathrm{~m}^{2}, 60 \mathrm{mg}$ ] was administered orally two times per day from after dinner on day 1 to after breakfast on day 15 , followed by a 7 -day rest. Maintenance chemotherapy with S-1 was permitted after discontinuing oxaliplatin, bevacizumab or both. Cycles were repeated until the criteria for withdrawal of the study treatment were met. In the event of disease progression after S-1 plus bevacizumab treatment, SOX plus bevacizumab could be reintroduced. The reintroduced SOX plus bevacizumab was continued until progression, unacceptable toxicity, or patient's wish to terminate the treatment.

\subsection{Evaluation of the Methods}

Objective tumor responses were evaluated according to the response evaluation criteria in solid tumors version 1.0 (RECICST v1.0) by each attending doctor. Adverse events were assessed according to the Common Terminology Criteria for Adverse Events version 3.0 (CTCAE v3.0).

Statistical analyses were performed using by the Statcel 2 software program (OMS, Saitama, Japan). The overall survival was calculated, using the Kaplan-Meier method, as the period from the date of bevacizumab treatment initiation until 
the date of death or until the last confirmed date of survival.

\section{Results}

Table 1 shows the characteristics of the 40 enrolled patients. The median age of the patients was 67.3 years (range 51 - 81 years). A total of 25 patients were male and 15 patients were female. ECOG performance status was 0 in all patients. The most common sites of metastasis were the liver, lung, lymph node, and peritoneum.

The median duration of treatment was 18.5 months (range, 2 - 58 months). SOX plus bevacizumab combination therapy was administered for a median of 3.93 cycles (range, 1 - 11 cycles). After discontinuation of oxaliplatin, 36 patients (90\%) continued with S-1 and bevacizumab combination therapy and received median 19.0 months (range, 2 - 53 months) (Figure 1). Two patients (5\%) continued with FOLFOX plus bevacizumab combination therapy.

At the final data cut-off date (31 Oct 2019), the median duration of follow-up was 36.0 months. 20 patients (50\%) had died of disease progression and 17 patients were still receiving study medication.

The analysis of efficacy is shown in Table 2. The median PFS was 15.0 months and median OS was 36.0 months. The response rate (RR: complete pus partial

Table 1. Patient characteristics.

\begin{tabular}{|c|c|c|}
\hline Characteristics & Number of patients & $(\%)$ \\
\hline \multicolumn{3}{|l|}{ Age (year) } \\
\hline Median (range) & 67.3 & $(51-81)$ \\
\hline \multicolumn{3}{|l|}{ Sex } \\
\hline Male & 25 & 62.5 \\
\hline Female & 15 & 37.5 \\
\hline \multicolumn{3}{|c|}{ ECOG performance status (PS) } \\
\hline 0 & 37 & 92.5 \\
\hline 1 & 3 & 7.5 \\
\hline \multicolumn{3}{|l|}{ Primary tumor site } \\
\hline Left & 27 & 67.5 \\
\hline Right & 13 & 32.5 \\
\hline \multicolumn{3}{|l|}{ Site of metastasis } \\
\hline Liver & 25 & 62.5 \\
\hline Lung & 16 & 40.0 \\
\hline Lymph node & 11 & 27.5 \\
\hline Peritoneum & 7 & 17.5 \\
\hline Local recurrence & 2 & 5 \\
\hline \multicolumn{3}{|l|}{ Line of treatment } \\
\hline 1 st & 40 & 100 \\
\hline \multicolumn{3}{|c|}{ Prior adjuvant therapy } \\
\hline No & 34 & 85 \\
\hline Yes & 8 & 15 \\
\hline
\end{tabular}


Table 2. Analysis of efficacy.

\begin{tabular}{|c|c|c|}
\hline \multicolumn{2}{|c|}{ Analysis of efficacy } & \\
\hline \multicolumn{3}{|c|}{ Endopoint } \\
\hline \multicolumn{2}{|c|}{ Median progressive-free survival, months } & $15.0 \mathrm{~m}$ \\
\hline \multicolumn{3}{|c|}{ 95\% Confidence interval } \\
\hline \multicolumn{2}{|c|}{ Median overall survival, months } & $36.0 \mathrm{~m}$ \\
\hline \multicolumn{2}{|c|}{ 95\% Confidence interval } & \\
\hline Response rate, \% & Number of patients & (\%) \\
\hline Complete response & 2 & 5.0 \\
\hline Partial response & 32 & 80 \\
\hline Stable disease & 3 & 7.5 \\
\hline Progressive disease & 3 & 7.5 \\
\hline $\mathrm{RR}$ & $85 \%$ & \\
\hline DCR & $92.5 \%$ & \\
\hline
\end{tabular}

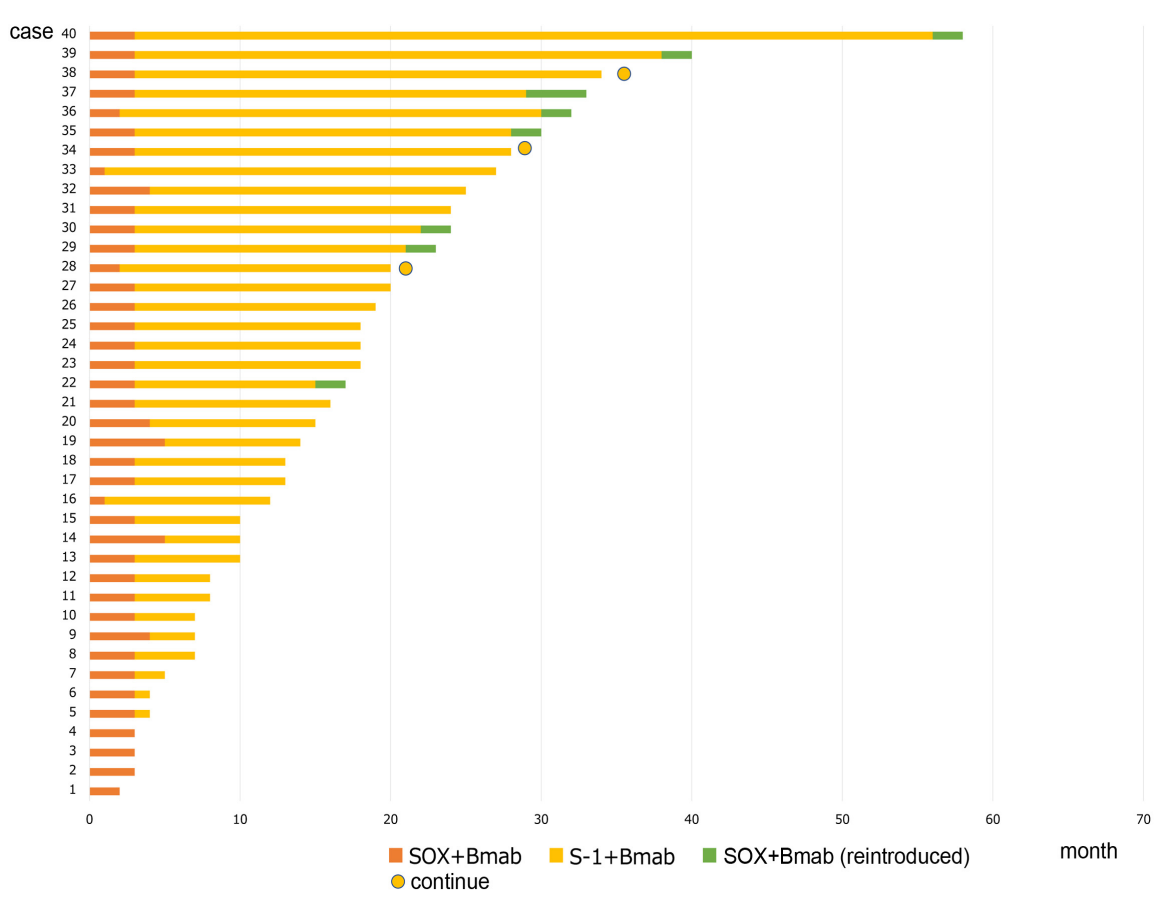

Figure 1. Period of treatment of SOX + bevacizumab, S-1 + bevacizumab, and reintroduced SOX + bevacizumab.

response) was $85.0 \%$, and the disease control rate (DCR: RR plus stable disease) was $92.5 \%$.

Table 3 shows the $2^{\text {nd }}$ line regimens used for patients treated with bevacizumab in the $1^{\text {st }}$ line regimen. It was revealed that $72.2 \%$ of the patients who were treated with bevacizumab in the $2^{\text {nd }}$ line regimen had been receiving bevacizumab continuously. $27.8 \%$ of the patients in the $2^{\text {nd }}$ line regimen had been receiving the combination chemotherapy with cetuximab or panitumumab.

Adverse events that occurred in all 40 patients are summarized in Table 4. 
Table 3. The second line regimens used for patients receiving SOX + bevacizumab as the first line treatment.

\begin{tabular}{cc}
\hline Regimen & No. of patients (\%), $\mathrm{n}=18$ \\
\hline FOLFIRI + bevacizumab & $5(27.7)$ \\
IRIS + bevacizumab & $4(22.2)$ \\
CAPIRI + bevacizumab & $4(22.2)$ \\
CPT-11 + cetuximab & $4(22.2)$ \\
FOLFIRI + panitumumab & $1(5.55)$ \\
\hline
\end{tabular}

Table 4. Incidence of common adverse events.

\begin{tabular}{ccccc}
\hline Adverse event & Grade 1 - & \multicolumn{3}{c}{ Grade 3 - } \\
\hline $\mathrm{n}=17$ & Number of patients & $(\%)$ & Number of patients & $(\%)$ \\
\hline Hypertension & 20 & 50 & 0 & 0 \\
Neurosensory toxicity & 20 & 50 & 0 & 0 \\
Anorexia & 13 & 32.5 & 5 & 12.5 \\
Fatigue & 18 & 45 & 9 & 22.5 \\
Hand-foot syndrome & 0 & 0 & 0 & 0 \\
Nausea/Vomitting & 15 & 37.5 & 1 & 2.5 \\
Diarrhea & 5 & 12.5 & 2 & 5 \\
Oral ulcer & 7 & 17.5 & 2 & 5 \\
Allergic reaction & 1 & 2.5 & 1 & 2.5 \\
Pigmentation & 12 & 30 & 0 & 0 \\
Neurtrophil count decreased & 12 & 30 & 1 & 2.5 \\
Pletelet count descreased & 5 & 12.5 & 0 & 0 \\
Proteinuria & 6 & 15 & 2 & 5 \\
\hline
\end{tabular}

The most common adverse events with SOX plus bevacizumab were hypertension $(50 \%)$, neurosensory toxicity $(50 \%)$, anorexia (32.5\%), fatigue (45\%), pigmentation (39\%), Neutrophil count decreased (30\%), and platelet count decreased (40\%). The most common grade $3 / 4$ adverse events were neurosensory toxicity (5\%) and fatigue (9\%). For patients receiving SOX plus bevacizumab, dose reduction was required for capecitabinein 6 patients $(15 \%)$; fatigue $(n=5)$ and diarrhea $(n=1)$. S-1 doses were reduced to $75 \%$ of starting dose in all 6 patients. Dose reductions were required for oxaliplatin in 0 patients $(0 \%)$ due to neurosensory toxicity, because of discontinuation of oxaliplatin, 36 patients (90\%) continued with S-1 and bevacizumab combination therapy.

\section{Discussion}

This study demonstrates the safety and efficacy of SOX with bevacizumab in combination with oxaliplatin $130 \mathrm{mg} / \mathrm{m}^{2}$ plus oral S-1 [40 - $60 \mathrm{mg}$, based on the body surface area (BSA): BSA $<1.25 \mathrm{~m}^{2}, 40 \mathrm{mg}$; $\mathrm{BSA} \geq 1.25 \mathrm{~m}^{2}$ to $<1.5 \mathrm{~m}^{2}, 50$ $\mathrm{mg} ; \mathrm{BSA} \geq 1.5 \mathrm{~m}^{2}, 60 \mathrm{mg}$ ] in Japanese patients. The previously reported primary 
analysis of the present study demonstrated that SOX plus bevacizumab is non-inferior to mFOLFOX6 plus bevacizumab in terms of PFS, the primary end point [10]. The MST was about 30 months and was similar in the SOX plus bevacizumab group and the mFOLFOX6 plus bevacizumab group. SOX plus bevacizumab is considered an effective regimen for first-line chemotherapy in patients with mCRC and can be used instead of mFOLFOX6 plus bevacizumab [9]. A phase III study in South Korea showed that SOX is non-inferior to CapeOX as first-line treatment for mCRC. 13 The incidence of HFS was lower in patients who received SOX (14\%) than in those who received CapeOX (31\%), whereas the RR was significantly higher in the SOX group (47\%) than that in the CapeOX group (36\%). This finding also suggests that SOX plus bevacizumab can contribute to maintaining a good quality of life among patients [11]. In this respect, SOX might be more advantageous to patients than CapeOX.

SOX plus bevacizumab can be given on an outpatient basis, with patients presenting at the hospital once every 3 weeks and does not require placement of a $\mathrm{CV}$ port. It is thus more convenient for patients than mFOLFOX6 plus bevacizumab. In addition, the incidence of grade 3 or higher neutropenia was distinctly lower with SOX plus bevacizumab than with mFOLFOX6 plus bevacizumab, making the former an easy-to-use regimen. The incidence of hand-foot syndrome (HFS) was lower in patients who received SOX (14\%) than in those who received Cape OX (31\%), whereas the RR was significantly higher in the SOX group (47\%) than that in the Cape OX group (36\%). This finding also suggests that SOX plus bevacizumab can contribute to maintaining a good quality of life among patients.

In recent studies with the uninterrupted FOLFOX regimen, the median PFS was in the range of 8.2 - 9.0 months, and severe neurotoxicity was observed in $18 \%-21 \%$ of patients [3] [12] [13].

In the OPTIMOX1 trial, which evaluated the efficacy of oxaliplatin stop-and-go strategy, PFS and DDC were 8.7 and 10.9 months, respectively. Grade 3 sensory neuropathy was observed in $13.3 \%$ of patients. Oxaliplatin was reintroduced in $40.1 \%$ of patients and objective response or disease stabilization was observed in $69.4 \%$ of these patients [14].

Phase II study evaluated S-1 on alternate days combined with bevacizumab as first-line treatment for elderly patients, age $\geq 75$ years, with metastatic colorectal cancer (J-SAVER). The median progression-free survival was 8.1 months. The median overall survival was 23.1 months. The response rate was $44 \%$, and the disease control rate was $88 \%$. S- 1 on alternate days combined with bevacizumab showed better tolerability and comparable survival compared with the results of similar studies [15].

Of significance is our novel data demonstrating the safety and efficacy of the international standard-dose S-1 and oxaliplatin (SOX) plus bevacizumab in Japanese patients. There were no fatal adverse events, and all complications were managed successfully using appropriate support care and drug cessation/dose reduction.

In our study, the median PFS was 15.0 months and median OS was 36.0 months. 
The response rate (RR: complete pus partial response) was $85.0 \%$, and the disease control rate (DCR: RR plus stable disease) was $92.5 \%$. The most common grade $3 / 4$ adverse events were neurosensory toxicity (5\%) and fatigue (9\%).

\section{Conclusion}

In conclusion, this study revealed that the survival benefit of S-1 and oxaliplatin (SOX) plus bevacizumab in Japanese patients with mCRC was like that observed in previous clinical trials. Therefore, S- 1 and oxaliplatin (SOX) plus bevacizumab can be considered as routine first-line treatment option for patients with mCRC.

\section{Conflicts of Interest}

The authors declare no conflicts of interest regarding the publication of this paper.

\section{References}

[1] Pirkin, D.M., Bray, F., Ferlay, J., et al. (2005) Global Cancer Statistics, 2002. CA: $A$ Cancer Journal for Clinicians, 55, 74-108. https://doi.org/10.3322/canjclin.55.2.74

[2] Kotake, K., Honjo, S., Sugihara, K., et al. (2003) Changes in Colorectal Cancer during a 20-Year Period: An Extended Report from the Multi-Institutional Registry of Large Bowel Cancer, Japan. Diseases of the Colon \& Rectum, 46, S32-S43.

[3] de Gramont, A., Figer, A., Seymour, M., et al. (2000) Leucovorin and Fluorouracil with or without Oxaliplatin as First-Line Treatment in Advanced Colorectal Cancer. Journal of Clinical Oncology, 18, 2938-2947. https://doi.org/10.1200/JCO.2000.18.16.2938

[4] Tournigand, C., Andre, T., Achille, E., et al. (2004) FOLFIRI Followed by FOLFOX6 or the Reverse Sequence in Advanced Colorectal Cancer: A Randomized GERCOR Study. Journal of Clinical Oncology, 22, 229-237. https://doi.org/10.1200/JCO.2004.05.113

[5] Cassidy, J., Tabernerro, J., Twelves, C., et al. (2004) XELOX (Capecitabine plus Oxaliplatin): Active First Line Therapy for Patients with Metastatic Colorectal Cancer. Journal of Clinical Oncology, 22, 2084-2091. https://doi.org/10.1200/JCO.2004.11.069

[6] Cassidy, J., Clarks, S., Diaz-Rubio, E., et al. (2008) Randomized Phase III Study of Capecitabine plus Oxaliplatin Compared with Fluorouracil/Folinic Acid plus Oxaliplatin as First-Line Therapy for Metastatic Colorectal Cancer. Journal of Clinical Oncology, 26, 2006-2012. https://doi.org/10.1200/JCO.2007.14.9898

[7] Uchima, Y., Nishii, T., Iseki, M., et al. (2014) Retrospective Analysis of Capecitabine and Oxaliplatin (XELOX) plus Bevacizumab as a First-Line Treatment for Japanese Patients with Metastatic Colorectal Cancer. Molecular and Clinical Oncology, 2, 134-138. https://doi.org/10.3892/mco.2013.205

[8] Satoh, T. and Sakata, Y. (2012) S-1 for the Treatment of Gastrointestinal Cancer. Expert Opinion on Pharmacotherapy, 13, 1943-1959. https://doi.org/10.1517/14656566.2012.709234

[9] Yamada, Y., Takahari, D., Matsumoto, H., et al. (2013) Leucovorin, Fluorouracil, and Oxaliplatin plus Bevacizumab versus S-1 and Oxaliplatin plus Bevacizumab in Patients with Metastatic Colorectal Cancer (SOFT): An Open-Label, Non-Inferiority, 
Randomised Phase 3 Trial. The Lancet Oncology, 14, 1278-1286. https://doi.org/10.1016/S1470-2045(13)70490-X

[10] Baba, H., Yamada, Y., Takahari, D., et al. (2017) S-1 and Oxaliplatin (SOX) plus Bevacizumab versus mFOLFOX6 plus Bevacizumab as First-Line Treatment for Patients with Metastatic Colorectal Cancer: Updated Overall Survival Analyses of the Open-Label, Non-Inferiority, Randomised Phase III: SOFT Study. ESMO Open, 2, e000135. https://doi.org/10.1136/esmoopen-2016-000135

[11] Hong, Y.S., Park, Y.S., Lim, H.Y., et al. (2012) S-1 plus Oxaliplatin versus Capecitabine plus Oxaliplatin for First-Line Treatment of Patients with Metastatic Colorectal Cancer: A Randomised, Noninferiority Phase 3 Trial. The Lancet Oncology, 13, 1125-1132. https://doi.org/10.1016/S1470-2045(12)70363-7

[12] Goldberg, R.M., Sargent, D.J., Morton, R.F., et al. (2004) A Randomized Controlled Trial of Fluorouracil plus Leucovorin, Irinotecan, and Oxaliplatin Combinations in Patients with Previously Untreated Metastatic Colorectal Cancer. Journal of Clinical Oncology, 22, 23-30. https://doi.org/10.1200/JCO.2004.09.046

[13] Goldstein, D., Mitchell, P., Michael, M., et al. (2005) Australian Experience of a Modified Schedule of FOLFOX with High Activity and Tolerability and Improved Convenience in Untreated Metastatic Colorectal Cancer Patients. British Journal of Cancer, 92, 832-837. https://doi.org/10.1038/sj.bjc.6602426

[14] Tournigand, C., Cervantes, A., Figer, A., et al. (2006) OPTIMOX1: A Randomised Study of FOLFOX4 or FOLFOX7 with Oxaliplatin in a Stop-and-Go Fashion in Advanced Colorectal Cancer-A GERCOR Study. Journal of Clinical Oncology, 24, 394-400. https://doi.org/10.1200/JCO.2005.03.0106

[15] Moriwaki, T., Sakai, Y., Ishida, H., et al. (2019) Phase II Study of S-1 on Alternate Days plus Bevacizumab in Patients Aged $\geq 75$ Years with Metastatic Colorectal Cancer (J-SAVER). International Journal of Clinical Oncology, 24, 1214-1222.

https://doi.org/10.1007/s10147-019-01465-3 